\title{
A Rare Case of Dynamic Popliteal Artery Occlusion After Gunshot Injury with Reconstitution of Flow in the Frog-leg Position
}

\author{
Franz Toro-Pape ${ }^{1}$, Boris Kumaev ${ }^{1}$, Matthew Jenson ${ }^{1}$, Jerry Matteo ${ }^{2}$ \\ 1. Department of Radiology, University of Florida College of Medicine, Jacksonville, USA 2. Department of \\ Interventional Radiology, University of Florida College of Medicine, Jacksonville, USA
}

Corresponding author: Franz Toro-Pape, franztoro@yahoo.com

\begin{abstract}
A 16-year-old male was transferred to our institution shortly after a gunshot injury to the right lower extremity. Physical examination was remarkable for two bullet entry points in the right posterior leg. A right lower extremity computed tomography angiogram (CTA) demonstrated a retained bullet fragment in the right popliteal fossa and a 10 centimeter $(\mathrm{cm})$ in length occlusion of the proximal peroneal artery with reconstitution of flow distally. A diagnostic angiogram of the right lower extremity with the patient's leg extended demonstrated lack of popliteal arterial flow immediately distal to the retained bullet fragment. Reconstitution of vascular flow was appreciated once the patient's leg was placed in the "frog-leg" position.
\end{abstract}

Categories: Cardiac/Thoracic/Vascular Surgery, Radiology, General Surgery

Keywords: popliteal artery entrapment, dynamic maneuvers, ct angiography, digital substraction angiography, gunshot injury, vascular injury, penetrating injury, popliteal artery injury, frog-leg position

\section{Introduction}

Penetrating gunshot injuries with or without concomitant vascular injuries are not uncommon at level 1 trauma centers, including our institution. The role of our interventional radiology department in their management and treatment has become increasingly important throughout the last two decades. The development and seemingly exponential improvement of minimally invasive transcatheter techniques over the last two decades have enabled the treatment of arterial injuries and potentially life-threatening bleeds with the use of minimally invasive angiography and intervention. At our institution, upper and lower extremity vascular injuries are frequently encountered clinical scenarios by our interventional radiologists, vascular surgeons, and general surgeons. Most of these are secondary to blunt or penetrating trauma resulting in arterial injury and frequently treated with balloon occlusion, transarterial embolization, coiling, and endovascular repair with or without stenting.

Received 04/20/2018 Review began 04/24/2018 Review ended 04/25/2018 Published 04/26/2018

\section{() Copyright 2018}

Toro-Pape et al. This is an open access article distributed under the terms of the Creative Commons Attribution License CC-BY 3.0., which permits unrestricted use, distribution, and reproduction in any medium, provided the original author and source are credited.
High-energy gunshot wounds carry the highest risk of vascular injury, followed by penetrating trauma with concomitant extremity fractures [1]. The inguinal region, medial compartment of the thigh, and popliteal fossa are considered high-risk locations for penetrating and blunt injuries in the lower extremities due to the superficial location of vessels in these locations. If not recognized and treated rapidly, traumatic peripheral vascular injuries may rapidly lead to loss of limb and life [2-3].

Although traumatic arterial injuries, as well as the effects of aging and mechanical stressors on the popliteal artery, have been well-documented in the literature [4], dynamic compression of the popliteal artery by a retained bullet fragment or other foreign bodies on diagnostic angiography is a rarely encountered clinical phenomenon and, to our knowledge, not yet reported in the literature. A focused literature search of the PubMed electronic database using the popliteal artery as a major topic in the Medical Subject Heading (MeSH) with additional MeSH subheading terminology, such as "digital subtraction angiography" (DSA), "angiography", "provocative DSA", "provocative maneuvers", "bullet", "ballistic", "angiography", "limb flexion", and "limb extension" was conducted, which did not yield a case similar to ours.

\section{Case Presentation}

The patient is a 16-year-old male without a significant past medical history who was transferred to our institution after a gunshot injury to the right lower extremity. On physical examination, two bullet entry points were evident at the right popliteal fossa and dorsal soft tissues of the distal right leg. Initial radiographs were negative for fractures or dislocation.

Computed tomography angiography (CTA) demonstrated a retained bullet fragment within the popliteal fossa abutting the dorsal aspect of the popliteal artery. An 8 millimeter $(\mathrm{mm})$ soft tissue density abutting the medial aspect of the popliteal artery was also identified, concerning for either a small pseudoaneurysm or short segment intramural hematoma. Streak artifact from the retained bullet precluded adequate assessment of this region. The peroneal artery demonstrated a 10 centimeter $(\mathrm{cm})$ occlusion $2.5 \mathrm{~cm}$ distal to 


\section{Cureus}

its origin but reconstituted distally at the level of the mid-tibia. The anterior and posterior tibial arteries were both normal in appearance and patent. The dorsalis pedis artery was unremarkable. There was subcutaneous emphysema throughout the deep and superficial posterior compartment of the knee and throughout the medial aspect of the leg.

The patient was taken to the interventional radiology suite and a right lower extremity diagnostic runoff angiogram was performed. Initial images obtained with the patient's leg held in extension demonstrated abrupt cutoff of the popliteal artery immediately adjacent to the bullet fragment (Figure 1). We then proceeded to reposition the patient's right leg in the "frog-leg position". A second diagnostic runoff angiogram was then performed demonstrating mild short segment narrowing of the popliteal artery immediately adjacent to the bullet fragment but with reconstitution of flow down to the level of the tibialperoneal trunk (Figure 2). The anterior and posterior tibial arteries demonstrated patency on both the extension and frog-leg positions. It was concluded that leg straightening/extension was contributing to extrinsic compression and subsequent dynamic occlusion of the popliteal artery secondary to the bullets close proximity to the vessel.

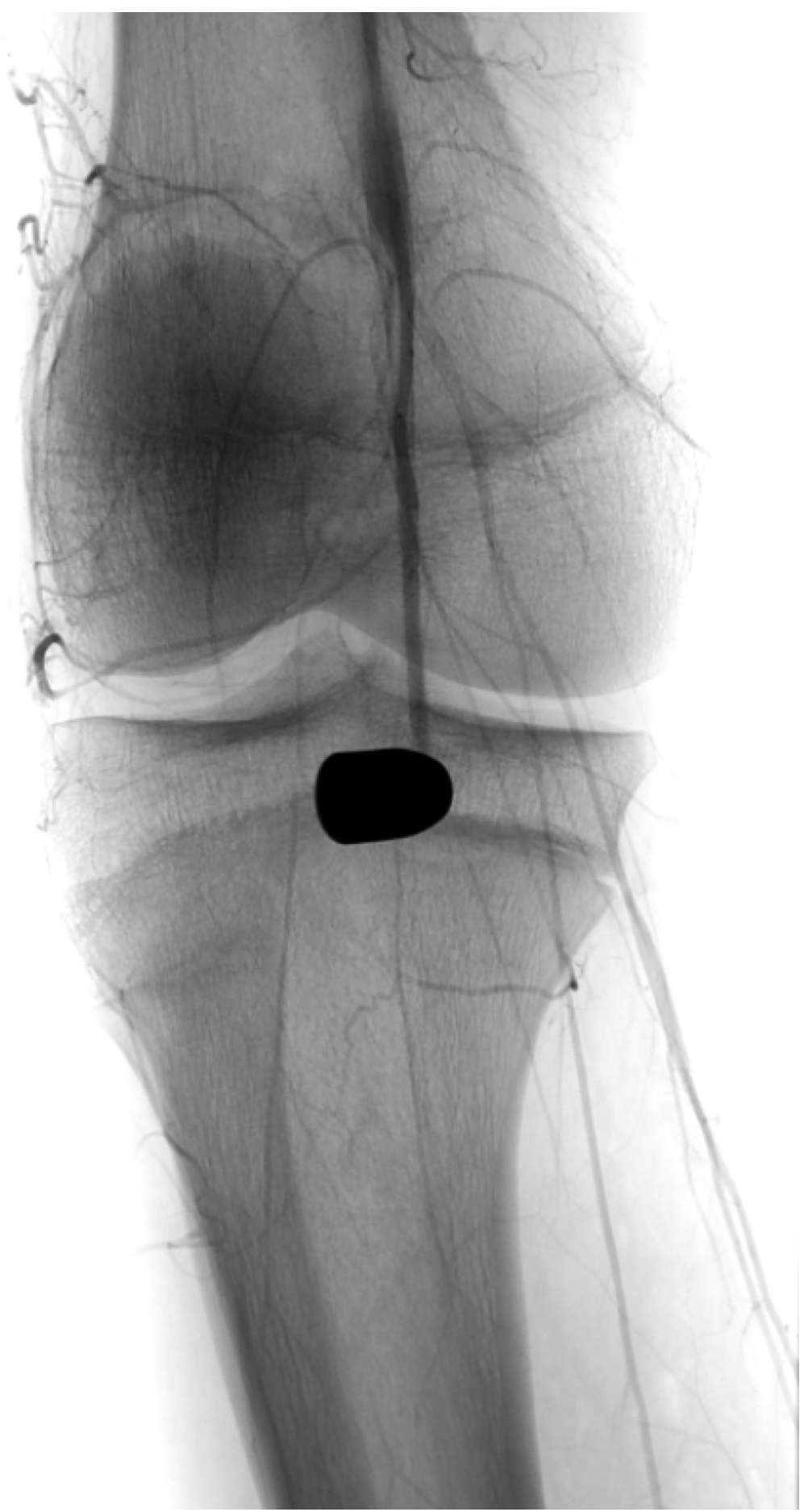




\section{Cureus}

FIGURE 1: Right lower extremity angiography at the level of the knee with the patient's leg held in extension

There is a retained bullet fragment in the popliteal fossa and abrupt cessation of vascular enhancement in the above the knee popliteal artery.

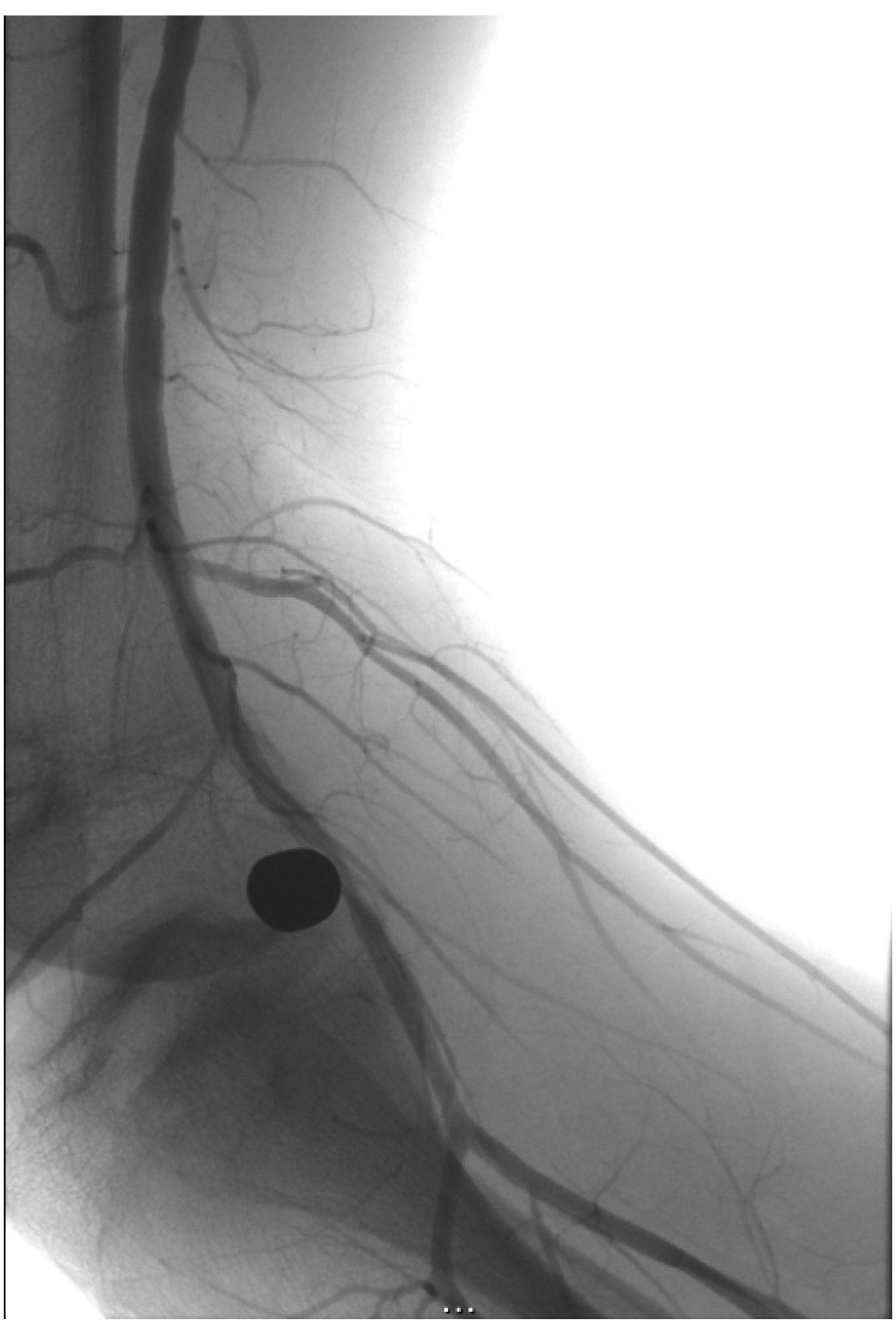

FIGURE 2: Right lower extremity angiography at the level of the knee with the patient's leg in the frog-leg position

There is reconstitution of flow within the popliteal artery down to the level of the tibial-peroneal trunk when the patient's leg was placed in the frog-leg position.

The patient was subsequently taken to the operating room for exploration and removal of the bullet fragment. An intraoperative angiogram was negative for popliteal artery vascular injury, and the anterior 


\section{Discussion}

The superficial femoral artery is a continuation of the common femoral artery after it gives off the deep femoral branches in the upper thigh. It continues its caudal course primarily throughout the medial aspect of the thigh, eventually becoming the popliteal artery past the adductor hiatus. In the lower extremities, arterial injuries below the adductor hiatus result in amputation more often than do injuries in any other site [5]. The management and the importance of quick diagnosis of lower extremity arterial injuries have been well-documented in the literature. Recognition of a vascular injury and expeditious transport to repair are imperative to avoid loss of limb and life.

Provocative maneuvers in order to evaluate the popliteal artery have been primarily described in cases of popliteal artery entrapment syndrome in patients undergoing CTA; however, to our knowledge, none have been described in the setting of trauma and DSA. A recent study highlighted the management and approach in the diagnosis of popliteal artery entrapment syndrome in a small sample of 15 patients. The workup included a two-part CTA first performed with the patient's knee in a neutral position, and a second with the knee hyperextended with active plantar flexion in order to contract the medial head of the gastrocnemius muscle. A total of 10 patients with mild stenosis and an additional two patients with severe stenosis during active plantar flexion were identified who had an otherwise negative exam when the knee was scanned in the neutral position [6]. This study highlights the importance of provocative maneuvers in the radiologic diagnosis of vascular entrapment syndromes.

A systematic review and meta-analysis evaluating the diagnostic accuracy of CTA in detecting arterial lesions in patients with suspected arterial injury of the upper or lower extremity showed that CTA is an accurate modality for evaluating arterial lesions in patients with extremity trauma and can replace digital subtraction angiography [7]. Although in full agreement that CTA is an invaluable diagnostic modality and essential for expeditious recognition of vascular injuries, DSA has the added benefit of intervention and extremity manipulation, as highlighted in our case. DSA is also less technically dependent, particularly in cases where the bolus may be deemed suboptimal for the evaluation of disease. This is of particular importance in the setting of trauma where time to diagnosis and subsequent intervention is of the essence.

\section{Conclusions}

Our case highlights the importance of dynamic maneuvers during diagnostic angiography in order to assess patency of potentially injured or occluded vessels when the extremity of interest is held in neutral position or fixed in the position of initial presentation. We recognize that provocative maneuvers are sometimes clinically not feasible due to concomitant secondary injuries and other related patient limitations frequently encountered in cases of penetrating or blunt extremity trauma.

\section{Additional Information \\ Disclosures}

Human subjects: Consent was obtained by all participants in this study. Conflicts of interest: In compliance with the ICMJE uniform disclosure form, all authors declare the following: Payment/services info: All authors have declared that no financial support was received from any organization for the submitted work. Financial relationships: All authors have declared that they have no financial relationships at present or within the previous three years with any organizations that might have an interest in the submitted work. Other relationships: All authors have declared that there are no other relationships or activities that could appear to have influenced the submitted work.

\section{References}

1. Miller-Thomas MM, West OC, Cohen AM: Diagnosing traumatic arterial injury in the extremities with CT angiography: pearls and pitfalls. Radiographics. 2005, 25:S133-42. 10.1148/rg.25si055511

2. Mollberg NM, Wise SR, Banipal S, et al.: Color-flow duplex screening for upper extremity proximity injuries: a low-yield strategy for therapeutic intervention. Ann Vasc Surg. 2013, 27:594-98. 10.1016/j.avsg.2012.10.006

3. de Mooij T, Duncan AA, Kakar S: Vascular injuries in the upper extremity in athletes . Hand Clin. 2015, 31:39-52. 10.1016/i.hcl.2014.09.004

4. Desyatova A, MacTaggart J, Romarowski R, et al.: Effect of aging on mechanical stresses, deformations, and hemodynamics in human femoropopliteal artery due to limb flexion. Biomech Model Mechanobiol. 2018, 17:181-89. 10.1007/s10237-017-0953-z

5. Snyder WH 3rd: Popliteal and shank arterial injury. Surg Clin North Am. 1988, 68:787-807. 10.1016/S00396109(16)44586-X

6. Papaioannou S, Tsitouridis K, Giataganas G, et al.: Evaluation of popliteal arteries with CT angiography in popliteal artery entrapment syndrome. Hippokratia. 2009, 13:32-37.

7. Jens S, Kerstens MK, Legemate DA, et al.: Diagnostic performance of computed tomography angiography in peripheral arterial injury due to trauma: a systematic review and meta-analysis. Eur J Vasc Endovasc Surg. 2013, 46:329-37. 10.1016/j.ejvs.2013.04.034 